\title{
Molecular vibrational states in the binary cold fission of ${ }^{252} \mathbf{C f}$
}

\author{
Ş Mişicu†, A Săndulescuł‡ and W Greinerł \\ $\dagger$ Institute for Nuclear Physics and Engineering, Bucharest-Măgurele, PO Box MG-6, \\ ROMANIA \\ $\ddagger$ Institut für Theoretische Physik der J W Goethe Universität, D-60054, Frankfurt am \\ Main, GERMANY
}

\begin{abstract}
We predict a molecular vibrational state in the cold binary fission of ${ }^{252} \mathrm{Cf}$ using a simple decay cluster model. The Hamiltonian of two even-even fragments in the pole-pole configuration is built in the same fashion as that for the dinuclear molecule formed in heavy-ions collisions. The interaction between the two fragments is described by the double-folding M3Y potential. The spectrum of the butterfly vibrations is derived and its dependence on fragments deformation and mass-assymetry is discussed. Some experimental implications are commented.
\end{abstract}

\section{Introduction}

Presently the cold fragmentation of heavy nuclei with masses ranging from $\mathrm{A} \approx 70$ to

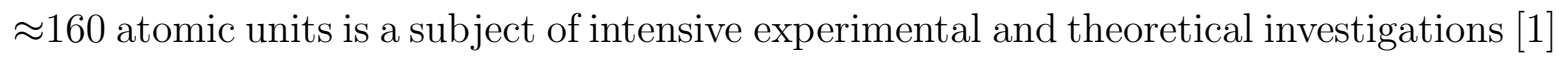
[2]. This phenomenon is an extension of the ordinary cluster radioactivity to the fission of heavy nuclei. In such processes the emerging fragments carry nearly no excitation energy (TXE) so that the kinetic energy (TKE) is close to the reaction $Q$-value. The binary cold neutronless fission of ${ }^{252} \mathrm{Cf}$ was recently observed using the multiple Gedetector arrays and the $\gamma-\gamma-\gamma$ coincidence technique [3]. In these experiments the even-even fragments were populating the lowest states with $I=0^{+}, 2^{+}, 4^{+}$of the $K=0^{+}$ground-state rotational band, which subsequently decayed through $\gamma$ emmision. The fragmentations involving three final nuclei have been also observed in these experiments. The two heavier fragments which have on the average 20 to $40 \mathrm{MeV}$ of total excitation energy (TXE) are usually accompanyed by a light charged particle. This third fragment is most likely to be an $\alpha$ particle. The relative transition probabilities to the $2^{+}, 4^{+}$and $6^{+}$excited states of the ground state rotational band and the $5^{-}$and $7^{-}$states of the octupole band are recorded for even-even heavy pairs (Ba$\mathrm{Zr}$ for example). Thus the individual fragments resulting from the cold fission process 
display a fine structure of rotational character mainly. A natural question then occur : Is it possible that the nascent fragments still bound together, close to the scission point, form a so called nuclear molecule similar to those produced by colliding heavy ions? The answer is positive if the potential between the fragments has pockets [4] as happens for isomeric states. On the other hand it is important to remind that the magic radioactivity consisting of the emission of heavy nuclei, such as ${ }^{14} \mathrm{C},{ }^{20} \mathrm{Ne},{ }^{28,30} \mathrm{Mg}$ and

${ }^{32} \mathrm{Si}$ nuclei, which has been predicted in the early eightees by Săndulescu, Poenaru and Greiner [5] can be portrayed as a particular example of nuclear molecules occuring in the fission process.

In the case of the binary cold fission such molecular excitations are more likely to occur when the two final nuclei are in the touching (nose-to-nose) configuration. The displayed collective spectrum should be analogous to that of a nuclear molecule formed by two colliding heavy ions sticking together for a short period of time and subjected to the interplay of Coulomb repulsion and nuclear attraction. In addition to the $\beta$ - and $\gamma$ - vibrational degrees of freedom of the individual nuclei, one finds dipole oscillations of the relative coordinate [6] and rotation-vibration modes of quadrupole nature like butterfly- and belly-dancer-type motions. In the present work we report results only for the butterfly modes (fig.1) We obtain their energy spectrum for several even-even splittings of ${ }^{252} \mathrm{Cf}$ employing the multipolar form of the M3Y potential for heavy deformed nuclei. This allows us to separate the radial from the angular variables in the Hamiltonian. Since the radial part of the M3Y potential does not contain pockets we disregard at once the molecular dipole oscillations. Moreover, since we do not expect major changes of the ground state deformations of the individual fragments during the cold fission process, the $\beta$ and $\gamma$ vibrations are excluded to.

\section{The Hamiltonian of the two fragments in the pole-pole configuration}

The classical form of the Hamiltonian for two interacting, quadrupole deformed nuclei is given by

$$
H=T+V\left(r, \alpha_{2 m}^{1}, \alpha_{2 m}^{2}\right)
$$

The kinetic energy reads

$$
T=\frac{1}{2} B_{1} \sum_{m} \dot{\alpha}_{2 m}^{1} \dot{\alpha}_{2-m}^{1}+\frac{1}{2} B_{2} \sum_{m} \dot{\alpha}_{2 m}^{2} \dot{\alpha}_{2-m}^{2}+\frac{1}{2} \mu \dot{\boldsymbol{r}}^{2}
$$

where the first two terms are giving the kinetic contribution from each fragment and the last one accounts for the relative kinetic energy. The double-folded deformed potential barrier was expressed in a previous paper [2] by performing a general multipole expansion 
of the potential

$$
V\left(\boldsymbol{R}, \Omega_{1}, \Omega_{2}\right)=\sum_{\lambda_{i}, \mu_{i}} V_{\lambda_{1} \lambda_{2} \lambda_{3}}^{\mu_{1} \mu_{2} \mu_{3}}(R) D_{\mu_{1} 0}^{\lambda_{1}}\left(\Omega_{1}\right) D_{\mu_{2} 0}^{\lambda_{2}}\left(\Omega_{2}\right) Y_{\lambda_{3} \mu_{3}}(\hat{R})
$$

In what follows we will limit ourselves to the case of monopole and quadrupole terms $\left(\lambda_{i}=0,2\right)$. As we mentioned before we consider that both fragments are aligned along the same symmetry axis and thus the above equation will have a more simple form

$$
V\left(R, \phi_{1}, \phi_{2}\right)=\sum_{\lambda_{i}=0,2} V_{\lambda_{1} \lambda_{2} \lambda_{3}}^{0} 00
$$

Because we consider the touching configuration (pole-pole), $\phi_{1}$ and $\phi_{2}$ are approximately related through the relation

$$
R_{1} \sin \phi_{1} \approx R_{2} \sin \phi_{2}
$$

where $R_{1}$ and $R_{2}$ are the radii along the symmetry axis for prolate nuclei $\left(R_{i}=\right.$ $\left.R_{0 i}\left(1+\sqrt{\frac{5}{\pi}} \beta_{0 i}\right)\right)$. For small inclinations angles $\left(\left|\phi_{1}\right|,\left|\phi_{2}\right| \ll 1\right)$ and taking into account that $\phi_{2}$ has opposite sign as $\phi_{1}$, the above equation reads

$$
\phi_{2} \simeq-\frac{R_{2}}{R_{1}} \phi_{1}
$$

The butterfly harmonic potential

$$
V_{\text {butt }}=\frac{1}{2} C_{\varepsilon} \varepsilon^{2}
$$

can be obtained from the M3Y potential by making a Taylor expansion in the variable $\varepsilon$ up to the second order, which eventually leads us to the expression of the stiffness

$$
\begin{aligned}
C_{\varepsilon}= & -3\left\{V_{202}\left(r_{c}\right)+V_{022}\left(r_{c}\right) \frac{R_{1}^{2}}{R_{2}^{2}}\right. \\
& \left.+\frac{1}{2}\left(1+\frac{R_{1}^{2}}{R_{2}^{2}}\right)\left(V_{220}\left(r_{c}\right)+V_{222}\left(r_{c}\right)+V_{224}\left(r_{c}\right)\right)\right\}
\end{aligned}
$$

This quantity is evaluated at the pole-pole configuration where $V\left(r_{c}\right)=V_{\text {butt }}$. It is worthwhile to notice that the monopole part of the potential does not contribute to the butterfly potential. Therefore the occurrence of the butterfly potential is strictly connected to the deformations of the fragments, i.e. only nuclear molecules with elongated shapes are able to experience such oscillations. It is also important to mention that since at the scission point the fragments have compact shapes, we can employ deformations close to the ground-state values. According to [7] the zeroth-order Hamilton operator for an asymmetric giant molecule reads

$$
\begin{aligned}
H_{0}^{\prime} & =\frac{\hbar^{2}}{2 \mu r_{0}^{2}}\left(L^{2}-L_{3}^{\prime 2}\right)+\frac{\hbar^{2}\left(L_{3}^{\prime 2}-\frac{1}{4}\right)}{6\left[B_{1} \beta_{01}^{2}+\frac{R_{1}^{2}}{R_{2}^{2}} B_{2} \beta_{02}^{2}\right] \varepsilon^{2}} \\
& -\frac{\hbar^{2}}{6\left[B_{1} \beta_{01}^{2}+\frac{R_{1}^{2}}{R_{2}^{2}} B_{2} \beta_{02}^{2}\right]} \frac{\partial^{2}}{\partial \varepsilon^{2}}+\frac{C_{\varepsilon}}{2} \varepsilon^{2}
\end{aligned}
$$


if we disregard the terms associated to the relative motion.

\section{The calculation of the butterfly energy spectrum}

The total wave function of the zeroth-order Hamiltonian, after symmetrization, is given by

$$
\left|I M K n_{\varepsilon}\right\rangle=\left[\frac{2 I+1}{16 \pi^{2}\left(1+\delta_{K 0}\right)}\right]^{\frac{1}{2}}\left(D_{M K}^{I \dagger}(\Omega)+(-)^{I} D_{M-K}^{I \dagger}(\Omega)\right) \chi_{K, n_{\varepsilon}}(\varepsilon)
$$

where [8]

$$
\chi_{K, n_{\varepsilon}}=\frac{\left\{\lambda^{l_{K}+\frac{3}{2}} \Gamma\left(l_{K}+\frac{3}{2}+n_{\varepsilon}\right)\right\}}{\left(n_{\varepsilon} !\right)^{1 / 2} \Gamma\left(l_{K}+\frac{3}{2}\right)}|\varepsilon|^{1 / 2} \varepsilon^{K / 2} e^{-\frac{1}{2} \lambda \varepsilon^{2}}{ }_{1} F_{1}\left(-n_{\varepsilon}, l_{K}+\frac{3}{2} ; \lambda \varepsilon^{2}\right)(13
$$

is the wave function describing the butterfly harmonic vibrations. The energy spectrum is then

$$
E_{I K n_{\varepsilon}}=\left(l_{K}+2 n_{\varepsilon}+\frac{3}{2}\right) \hbar \omega_{\varepsilon}+\left(I(I+1)-K^{2}\right) \frac{\hbar^{2}}{2 \mu r_{0}^{2}}
$$

with $n_{\varepsilon}=0,1,2, \ldots ; K=0,2,4, \ldots ; I=0,2,4, \ldots$ if $K=0$ and $I=K, K+1, K+2, \ldots$ if $K \neq 0$. The butterfly oscillation frequency is

$$
\omega_{\varepsilon}=\sqrt{\frac{C_{\varepsilon}}{2 \mathcal{J}_{12}}}
$$

with $\mathcal{J}_{12}=3\left[B_{1} \beta_{01}^{2}+\frac{R_{1}^{2}}{R_{2}^{2}} B_{2} \beta_{02}^{2}\right]$ being the inertia parameter of the nuclear molecule. We notice that this model works only for even-even splittings of ${ }^{252} \mathrm{Cf}$. In order to extend the calculations to the odd-odd splittings the particle-core coupling must pe appropriately included in the Hamiltionian. We will address this question in a forthcoming paper In Table I we list the zero-energy $\hbar \omega_{\varepsilon}$ for nuclear molecule configurations corresponding to even-even splitings of ${ }^{252} \mathrm{Cf}$. It is important to substantiate once again the importance of fragments deformation in the excitation of these states. When both fragments are well deformed the first excited state $\left(\approx 2 \hbar \omega_{\varepsilon}\right)$ is lying at low energy. If one of the emitted fragments is nearly spherical the location is shifted up in energy up to 10 times or more. Therefore it is expected to observe these states for binary cold fission channels with fragments having large deformations. On the other hand, resuming the calculations from Table I, but this time with the same values for the deformations of the light and heavy fragments, we arrive at the conclusion that the energy of these vibrations depends only sligthly on the mass assymetry. 
Table 1. The value of butterfly zero-energy $\hbar \omega_{\varepsilon}$ for different even-even splittings of ${ }^{252} \mathrm{Cf}$

\begin{tabular}{|c|c|c|c|c|}
\hline Light fragment & $\varepsilon_{L}$ & Heavy fragment & $\varepsilon_{H}$ & $\hbar \omega_{\varepsilon}(\mathrm{KeV})$ \\
\hline${ }^{94} \mathrm{Kr}$ & 0.270 & ${ }^{158} \mathrm{Sm}$ & 0.300 & 517.88 \\
\hline${ }^{94} \mathrm{Sr}$ & 0.215 & ${ }^{158} \mathrm{Nd}$ & 0.295 & 636.42 \\
\hline${ }^{96} \mathrm{Sr}$ & 0.240 & ${ }^{156} \mathrm{Nd}$ & 0.320 & 607.68 \\
\hline${ }^{98} \mathrm{Sr}$ & 0.330 & ${ }^{154} \mathrm{Nd}$ & 0.230 & 609.36 \\
\hline${ }^{98} \mathrm{Zr}$ & 0.255 & ${ }^{154} \mathrm{Ce}$ & 0.283 & 603.70 \\
\hline${ }^{100} \mathrm{Sr}$ & 0.350 & ${ }^{152} \mathrm{Nd}$ & 0.250 & 509.43 \\
\hline${ }^{100} \mathrm{Zr}$ & 0.273 & ${ }^{152} \mathrm{Ce}$ & 0.267 & 623.05 \\
\hline${ }^{102} \mathrm{Zr}$ & 0.340 & ${ }^{150} \mathrm{Ce}$ & 0.200 & 767.60 \\
\hline${ }^{104} \mathrm{Mo}$ & 0.315 & ${ }^{148} \mathrm{Ba}$ & 0.245 & 597.80 \\
\hline${ }^{104} \mathrm{Zr}$ & 0.300 & ${ }^{140} \mathrm{Ce}$ & 0.210 & 767.60 \\
\hline${ }^{106} \mathrm{Mo}$ & 0.245 & ${ }^{146} \mathrm{Ba}$ & 0.200 & 1074.16 \\
\hline${ }^{106} \mathrm{Zr}$ & 0.330 & ${ }^{146} \mathrm{Ce}$ & 0.170 & 815.05 \\
\hline${ }^{108} \mathrm{Mo}$ & 0.245 & ${ }^{144} \mathrm{Ba}$ & 0.198 & 1088.19 \\
\hline${ }^{108} \mathrm{Ru}$ & 0.300 & ${ }^{144} \mathrm{Xe}$ & 0.200 & 805.73 \\
\hline${ }^{110} \mathrm{Mo}$ & 0.240 & ${ }^{142} \mathrm{Ba}$ & 0.150 & 1421.21 \\
\hline${ }^{112} \mathrm{Ru}$ & 0.220 & ${ }^{140} \mathrm{Xe}$ & 0.120 & 2051.47 \\
\hline${ }^{114} \mathrm{Ru}$ & 0.180 & ${ }^{138} \mathrm{Xe}$ & 0.060 & 3664.41 \\
\hline${ }^{114} \mathrm{Pd}$ & 0.150 & ${ }^{138} \mathrm{Te}$ & 0.140 & 2863.09 \\
\hline${ }^{116} \mathrm{Pd}$ & 0.140 & ${ }^{136} \mathrm{Te}$ & 0.060 & 4956.01 \\
\hline${ }^{116} \mathrm{Ru}$ & 0.150 & ${ }^{136} \mathrm{Xe}$ & -0.020 & 4839.04 \\
\hline${ }^{118} \mathrm{Pd}$ & 0.100 & ${ }^{134} \mathrm{Te}$ & 0.000 & 7736.05 \\
\hline${ }^{120} \mathrm{Cd}$ & 0.030 & ${ }^{132} \mathrm{Sn}$ & -0.010 & 14497.94 \\
\hline${ }^{120} \mathrm{Pd}$ & 0.110 & ${ }^{132} \mathrm{Te}$ & -0.005 & 4076.04 \\
\hline${ }^{122} \mathrm{Pd}$ & 0.008 & ${ }^{108} \mathrm{Te}$ & 0.000 & 9213.6 .1 \\
\hline
\end{tabular}




\section{Concluding remarks}

The recent measurement of the double fine structure in the cold fission of ${ }^{252} \mathrm{Cf}$ opened the interest to search for possible molecular states in the fragmentation process where two or more nuclei are involved. The believe on the existence of such collective excitations is supported by the fact that cold fission is just a natural extension of cluster radioactivity to heavy nuclei. In this paper we predicted a molecular vibrational mode for the cold fission of ${ }^{252} \mathrm{Cf}$ in two fragments with axial symmetric deformation. Geometrically this mode may be pictured as in-phase rotational vibrations of the two fragments with respect to an axis perpendicular to their symmetry axis which passes through the touching point. The magnitude of the butterfly quantum energy depends strongly on the ground state deformations of both fragments. Concerning the possibility to observe $\gamma$ transitions from butterfly-type molecular states we make the observation that such a project is feasible. The large bulk of experimental data provided by the Gamma sphere facility from ORNL on the $\gamma$ decay transitions from low-lying states of individual fragments will turn out to be the corner stone in the search of similar transitions but of nuclear molecular nature. As for the future we intend to extend these calculations in order to encompass also the odd-odd splittings and to study the influence

of higher multipolarity deformations like octupole and hexadecupole on the molecular vibrational spectra.

\section{References}

[1] Knitter H-H, Hambsch F J and Budtz-Jorgensen C 1993 Nucl. Phys. A 554209

[2] Săndulescu A, Florescu A, Cârstoiu F, Greiner W, Hamiltion J H, Ramayya A V and Babu B R S 1996 Phys. Rev. C 54258

[3] Ter-Akopian G M et al 1994 Phys. Rev. Lett. 731477

[4] Greiner W, Park Jae Y, Scheid W 1995 Nuclear Molecules World Scientific, Singapore

[5] Săndulescu, Poenaru D, Greiner W 1980 Sov. J. Part. Nucl. 11528

[6] Iachello F 1981 Phys. Rev. C 232778

[7] Hess P O and Greiner W 1984 Nuovo Cimento 83A 76

[8] Eisenberg J M and Greiner W 1975 Nuclear Theory I North-Holland, Amsterdam 
Figure captions

Figure 1. Butterfly-type oscillations of a nuclear molecule 